Pesq. Vet. Bras. 30(10):861-867, outubro 2010

\title{
Desenvolvimento de um modelo experimental de hemodiálise em cães ${ }^{1}$
}

\author{
Andre M.C. Meneses ${ }^{2}$, Jacqueline C.T. Caramori ${ }^{3}$, João R.A.C. Brant ${ }^{4}$, \\ Raquel C. Gonçalves ${ }^{5}$, Nazaré F. de Souza ${ }^{2}$, Carla C.G. de Moraes ${ }^{6}$, Regina \\ K. Takahira ${ }^{7}$ e Pasqual Barretti ${ }^{3}$
}

\begin{abstract}
Meneses A.M.C., Caramori J.C.T., Brant J.R.A.C., Gonçalves R.C., Souza N.F., Moraes C.C.G., Takahira R.K. \& Barretti P. 2010. [Development of a dog model of hemodialysis.] Desenvolvimento de um modelo experimental de hemodiálise em cães. Pesquisa Veterinária Brasileira 30(10):861-867. Instituto da Saúde e Produção Animal, Universidade Federal Rural da Amazônia, Av. Presidente Tancredo Neves 2501, Montese, Cx. Postal 917, Belém, PA 66077-530, Brazil. E-mail: andre.meneses@ufra.edu.br

To develop a model of hemodialysis (HD), 18 healthy dogs, without a defined breed, males, weighing 7-14 kg, were studied. A double lumen catheter was inserted into the jugular vein for vascular access. HD sessions, totalizing 5 for each dog, were performed with a proportional HD machine, controlled isovolemic ultrafiltration, standard dialysis solution and bicarbonate buffer. Sodium profile and sodium heparin were used. During HD sessions the dogs were kept anesthetized (levomepromazine and propofol). Hematological and biochemistry data, blood gas analysis, systemic arterial blood pressure, and activated clotting time were evaluated. Serum biochemistry e blood gas analysis showed, respectively, sodium and $\mathrm{SO}_{2}$ maintenance. Systemic blood pressure kept sustained during HD sessions. It could be concluded that it was possible to develop a HD dog model that is a safe and viable technique to be used in chronic renal failure patients.
\end{abstract}

INDEX TERMS: Hemodialysis, experimental model, dogs.

RESUMO.- Com o objetivo de desenvolver um modelo de hemodiálise (HD) em cães, foram estudados 18 animais, sem raça definida, machos, clinicamente sadios, com peso corporal variando entre sete e $14 \mathrm{~kg}$. O acesso vascular foi obtido através de implantação do cateter de duplo lúmen

\footnotetext{
${ }^{1}$ Recebido em 25 de março de 2010.

Aceito para publicação em 3 de junho de 2010.

2 Instituto da Saúde e Produção Animal, Universidade Federal Rural da Amazônia, Av. Presidente Tancredo Neves 2501, Montese, Cx. Postal 917, Belém, PA 66077-530, Brasil. *Autor para correspondência: andre.meneses@ufra.edu.br

${ }^{3}$ Departamento de Clínica Médica, Faculdade de Medicina, Universidade Estadual Paulista (Unesp), Distrito de Rubião Júnior $s / n$, Botucatu, SP 18618-970, Brasil

${ }^{4}$ Médico Veterinário Autônomo, SHLS Q.716, Conj.L, BI.2, Asa Sul, Brasília, DF 70100-000, Brasil

${ }^{5}$ Curso de Medicina Veterinária, Centro de Ciências Humanas, da Saúde, Exatas e Tecnológicas, Unopar, Campus Universitário de Arapongas, PR $218 \mathrm{Km} \mathrm{1,} \mathrm{Jd.} \mathrm{Universitário,} \mathrm{Cx.} \mathrm{Postal} \mathrm{560,} \mathrm{Arapongas,}$ PR 86702-670, Brasil.

${ }^{6}$ Faculdade de Medicina Veterinária, Universidade Federal do Pará, Campus Castanhal, Rua Maximino Porpino 1000, Bairro Pirapora, Castanhal, PA 68743-000, Brasil.

${ }^{7}$ Departamento de Clínica Veterinária, Faculdade de Medicina Veterinária e Zootecnia, Unesp, Botucatu, SP.
}

em veia jugular externa. As sessões de HD, em número de cinco por animal, com até três horas de duração, foram realizadas em hemodialisadora de sistema proporcional com ultrafiltração (UF) controlada, com solução dialisante padrão e tampão bicarbonato. A UF foi ajustada para HD isovolêmica, utilizou-se perfil de sódio, e para anticoagulação heparina sódica. Os animais foram mantidos anestesiados com cloridrato de levomepromazina e propofol. Foram avaliados dados hematológicos, bioquímicos, hemogasometria, pressão arterial sistêmica e tempo de coagulação ativado. Foi observada diminuição do número global de hemácias, volume globular, hemoglobina e leucócitos. Em relação aos exames bioquímicos, houve manutenção nos níveis de sódio sérico, e quanto à hemogasometria, a manutenção da $\mathrm{SO}_{2}$. A pressão arterial sistêmica manteve-se constante. Os resultados obtidos no presente trabalho permitiram concluir que foi possível o desenvolvimento do modelo proposto e mostrou que a HD em cães é um método viável e seguro, que poderá contribuir para o tratamento clínico da insuficiência renal nesta espécie.

TERMOS DE INDEXAÇÃO: Hemodiálise, modelo experimental, cães. 


\section{INTRODUÇÃO}

O rim é o principal órgão envolvido na manutenção do equilíbrio hidroeletrolítico do organismo. Além de manter a homeostase, exerce também importante atividade de síntese, sendo capaz de produzir diversos hormônios e substâncias vasoativas com efeitos endócrinos e parácrinos (Boim et al. 2000). A importância dos rins torna-se evidente quando se observam as consequências da perda da função renal. O indivíduo em insuficiência renal retém, progressivamente, produtos do metabolismo nitrogenado, como a ureia; acumula líquidos sob a forma de edema; perde capacidade de diluir e concentrar urina; torna-se incapaz de regular o equilíbrio ácido-básico e de manter o balanço e os níveis plasmáticos de eletrólitos, tais como sódio, potássio, magnésio, cálcio e fósforo (Yu et al. 2000).

Em pacientes com uremia grave, a excreção renal pode ser parcialmente substituída através da hemodiálise (HD), método no qual um "rim artificial" é utilizado para corrigir os desequilíbrios da composição e volume do fluido corpóreo e ainda eliminar toxinas acumuladas. A HD tem grande aplicação terapêutica, utilizando métodos de difusão e ultrafiltração, para promover a remoção de solutos tóxicos, obter a redução do volume e corrigir a composição dos fluidos corpóreos, alterados pela função renal diminuída (Cowgill \& Langston 1996).

As principais indicações para a instituição da HD em pequenos animais são os casos de insuficiência renal aguda (IRA), estágios finais da insuficiência renal crônica (IRC), envenenamentos agudos, intoxicações e hipervolemia (Cowgill \& Langston 1996).

A anticoagulação foi realizada de forma sistêmica, utilizando-se, para tanto, a heparina sódica. Segundo Cowgill \& Langstone (1996), sua utilização previne a coagulação e adesão plaquetária no dialisador e circuito extracorpóreo, visto que esses eventos reduzem a área de superfície para diálise e contribuem para a perda de sangue durante o tratamento. A dose de heparina a ser utilizada varia de 50 a $100 \mathrm{U} / \mathrm{kg} / \mathrm{IV}$, mantendo-se em infusão constante na dose de 200-1200 U/h (Cowgill 1995).

Segundo Langston et al. 1996, a HD tem sido realizada com sucesso em cães, utilizando técnica descrita por Cowgill (1995). Entretanto, caso não seja possível a realização da HD, para aqueles pacientes acometidos de doenças renais agudas ou crônicas, o prognóstico é desfavorável, sendo o óbito quase inevitável (Cowgill \& Langston 1996).

A realização do presente estudo justifica-se, do ponto de vista clínico, pelo crescente número de pacientes veterinários com insuficiência renal, fato este que reforça a grande necessidade da qualificação de profissionais para aplicação de técnicas de tratamento que aumentem a sobrevida destes pacientes. Por outro lado, o desenvolvimento deste modelo experimental permitirá estudos posteriores e a realização de novas pesquisas, com aplicações em pacientes veterinários e humanos.

\section{MATERIAL E MÉTODOS}

O presente estudo foi realizado no Laboratório Experimental do Departamento de Clínica Médica da Faculdade de Medicina,
Universidade Estadual Paulista (Unesp), Campus de Botucatu, tendo sido aprovado junto a Comissão de Ética na Experimentação Animal da Faculdade de Medicina da Unesp-Botucatu, protocolo $\mathrm{n}^{\circ} 155$ de 27 de dezembro de 2000, de acordo com os princípios Éticos na Experimentação Animal adotado pelo Colégio Brasileiro de Experimentação Animal (Cobea).

Foram utilizados 18 cães, sem predisposição racial, adultos, machos, com peso corporal variando entre 7 e $14 \mathrm{~kg}$, clinicamente sadios, provenientes do Canil do Biotério Central da Unesp-Botucatu. Dois dias antes do início do estudo, os animais foram submetidos à implantação do cateter de duplo lúmen, segundo técnica descrita por Meneses et al. (2002) e avaliação radiográfica para verificar o correto posicionamento do mesmo. No dia das sessões de HD, os animais foram submetidos a jejum alimentar de 12 horas e hídrico de 2 horas, sendo então pré-anestesiados com levomepromazina (1mg/ $\mathrm{kg} / \mathrm{IV}$ ), utilizando-se o propofol como anestesia geral intravenosa (indução: $5 \mathrm{mg} / \mathrm{kg} / \mathrm{lV}$; manutenção: $0,2-0,4 \mathrm{mg} / \mathrm{kg} /$ IV/min, diluído em solução de glicose 5\%).

Foi utilizado aparelho de HD de sistema proporcional ${ }^{8}$, com módulo de ultrafiltração controlada, o qual foi calibrado para as necessidades do paciente. Empregou-se dialisador de polissulfona com área de superfície $0,4 \mathrm{~m}^{2} \mathrm{e}$ volume de preenchimento $28 \mathrm{~mL}^{9}$. O fluxo sanguíneo extracorpóreo foi restrito a $3-5 \mathrm{~mL} / \mathrm{kg} / \mathrm{min}$ nos dois tratamentos iniciais, para que se pudesse evitar excessivo clearence de ureia e desequilíbrio osmótico; a partir da terceira sessão houve aumento progressivo para $10-15 \mathrm{~mL} / \mathrm{kg} / \mathrm{min}$, com duração de até 180 minutos cada, num total de cinco sessões.

A anticoagulação foi realizada com heparina sódica, em protocolo com dose inicial de $100 \mathrm{U} / \mathrm{kg} / \mathrm{IV}$, repetindo-se 25U/ $\mathrm{kg} / \mathrm{lV}$ na primeira e segunda hora da sessão (Meneses 2003). Foi determinado o tempo de coagulação ativada (TCA) com monitor MCA $2000^{10}$, nos momentos 0, 5, 120 e 180 minutos do procedimento. Vale ressaltar que o TCA basal foi determinado antes que os animais iniciassem as sessões de HD. Determinou-se o volume de preenchimento do hemodialisador após cada sessão para se verificar a possibilidade de reutilização do referido capilar. Para tal preenchia-se o filtro com solução salina e, com auxílio de um jato de ar, esvaziava-se o conteúdo do mesmo em um Becker milimetrado.

Utilizando-se venopunção jugular, foram colhidas amostras pra realização de hemograma (número de hemácias $(\mathrm{mL})$, hemoglobina (g/dL), volume globular (\%), proteína total (plasma) $(\mathrm{g} / \mathrm{dL}))$, contagem de plaquetas $(\mathrm{mL})$; dosagens séricas de ureia $(\mathrm{mg} / \%)$, creatinina $(\mathrm{mg} / \%)$, sódio $(\mathrm{mmol} / \mathrm{L})$, potássio $(\mathrm{mmol} / \mathrm{L})$, glicose ( $\mathrm{mg} / \%)$, proteína total $(\mathrm{g} / \%)$, albumina ( $\mathrm{g} / \%)$, e globulina $(\mathrm{g} / \%)$, nos momentos pré $(\mathrm{PRH})$ e pós- hemodiálise $(\mathrm{POH})$.

Por meio de punção da artéria femural, colheu-se sangue para hemogasometria, anaerobicamente, com auxílio de seringa plástica previamente heparinizada, sendo a amostra acondicionada em recipiente isotérmico a $4^{\circ} \mathrm{C}$ até o momento da análise hemogasométrica (até $3 \mathrm{~h}$ após a colheita). Foram determinados $\mathrm{pH}$, pressão parcial de $\mathrm{O}_{2}\left(\mathrm{PO}_{2}\right)$, pressão parcial

\footnotetext{
8 Tina ${ }^{\circledR}$, Baxter Healthcare - Brasil, Av. Alfredo Egídio de Souza Aranha 100, Chácara Santo Antônio, São Paulo, SP.

9 F3®, Fresenius Medical Care, Rua Amoreira 891, Centro, Jaguariúna, SP.

10 MCA2000®, Fundação Adib Jatene, Av. Dr. Dante Pazzanese 500 , Vila Mariana, São Paulo, SP.

${ }^{11}$ Gould RS-3200®, Gould Electronic Inc., 8333 Rockside Rd, Valley View, Cleveland, OH, USA.
} 
de $\mathrm{CO}_{2}\left(\mathrm{PCO}_{2}\right)$, concentração de $\mathrm{CO}_{2}$ total, bicarbonato $\left(\mathrm{HCO}_{3}\right)$, e saturação de $\mathrm{O}_{2}$ nas hemoglobinas $\left(\mathrm{SO}_{2}\right)$, nos momentos M0, M90 e M180.

As pressões arteriais foram mensuradas pelo aparelho Gould RS-3200 ${ }^{11}$ utilizando-se o método invasivo, através da cateterização arterial (femural ou metatársica), nos momentos pré- HD, 5, 30, 60, 90, 120, 150 e 180 minutos. Os resultados da pressão arterial sistólica (PAS) e pressão arterial diastólica (PAD) foram obtidos através de gráficos e expressos em $\mathrm{mmHg}$.

Imediatamente após a realização das sessões de HD, na área onde estava inserido o cateter de duplo lúmen instalado na veia jugular externa foi realizada antissepsia do orifício de saída do cateter e das vias arterial e venosa do cateter. Posteriormente as vias foram lavadas com solução fisiológica 0,9\%, e preenchidas com heparina sódica $(0,7 \mathrm{~mL}$ da via arterial e $0,8 \mathrm{~mL}$ da via venosa) para evitar a presença de coágulos nas vias, fato este que diminuiria a perviedade do cateter e até poderia impossibilitar sua utilização. Foi colocado curativo no local para evitar que os animais retirassem acidentalmente o cateter.

Os dados foram inicialmente analisados quanto à aderência à curva de Gauss, pelo teste de distância K-S, sendo os paramétricos (Wichern \& Johnson 1992) expressos como média e desvio padrão e os não paramétricos (Siegel \& Castellan Jr 1988) como mediana. Para os dados paramétricos, a comparação entre os diferentes momentos foi realizada, utilizando-se a análise de variância para medidas repetidas e o pósteste de Newman-Kleuls, enquanto para os não paramétricos utilizou-se a prova não paramétrica de Friedman para medidas repetidas e o pós-teste de Newman-Keuls. O nível de significância foi de $5 \%$.

\section{RESULTADOS}

Os resultados relativos às determinações do TCA (segundos) nos momentos M5, M60, M120 e M180, expressos em mediana estão representados no Quadro 1. A média do TCA basal foi de 95,42 segundos. A análise estatística mostrou que as medianas nos momentos M5, M60 e M120 não apresentaram diferença estatística entre si e foram maiores que $\mathrm{M} 180(\mathrm{P}<0,001)$.

Em relação ao volume de preenchimento do dialisador utilizado e o reúso, observou-se que a média foi de $23 \pm 6,66$, o que caracteriza uma perda menor que $20 \%$ do priming inicial.

A análise estatística mostrou que houve diferença significante entre as medianas dos momentos pré (PRH) e pós-hemodiálise $(\mathrm{POH})$, quando avaliadas contagem glo-

Quadro 1. Mediana, semi-amplitude interquantílica e valores mínimo e máximo do tempo de coagulação ativada (TCA) nos momentos 5, 60, 120 e 180 minutos de procedimento hemodialítico e resultado do teste estatístico da comparação de momentos

\begin{tabular}{ccccc}
\hline Momentos & \multicolumn{4}{c}{ Medida descritiva } \\
\cline { 2 - 5 } de avaliação & Mediana & $\begin{array}{c}\text { Semi-amplitude } \\
\text { interquantílica }\end{array}$ & $\begin{array}{c}\text { Valor } \\
\text { máximo }\end{array}$ & $\begin{array}{c}\text { Valor } \\
\text { mínimo }\end{array}$ \\
\hline 5 Minutos & 328,60 & 8,75 & 1108,6 & 227 \\
60 Minutos & 229,30 & 7 & 666,2 & 149 \\
120 Minutos & 232,10 & 6 & 278,8 & 125,6 \\
180 Minutos & 189,80 & 10,75 & 273,2 & 142 \\
\hline
\end{tabular}

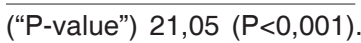

bal de hemácias $(P<0,001)$, volume globular $(P<0,001)$ e número total de plaquetas $(P<0,001)$, sendo que, em todas as variáveis os valores de $\mathrm{PRH}$ foram maiores que os de $\mathrm{POH}$, assim como representado no Quadro 2.

Os valores das médias de hemoglobina, proteína total (plasma) e número de leucócitos, apresentaram diferença estatística, respectivamente, com $\mathrm{P}<0,001, \mathrm{P}<0,001$ e $\mathrm{P}<0,005$, assim como representado no Quadro 3.

Todas as determinações bioquímicas foram avaliadas nos momentos $\mathrm{PRH}$ e $\mathrm{POH}$. O Quadro 4 representa as determinações séricas de magnésio, glicose, proteína, albumina. Os valores de magnésio não apresentaram, estatisticamente, diferença entre os dois momentos estudados $(P>0,05)$. Quanto à glicose sérica houve diferença estatística entre os dois momentos, sendo que os valores $\mathrm{PRH}$ foram maiores que os $\mathrm{POH}(\mathrm{P}<0,001)$. Estatisticamente, houve diferença entre os momentos $\mathrm{PRH}$ e $\mathrm{POH}$ referentes aos valores de proteína total $(P<0,05)$, albumina $(P<0,001)$ e globulina $(P<0,01)$, sendo que os $P R H$ foram maiores que $\mathrm{POH}$.

O Quadro 5 representa as determinações séricas de ureia, creatinina, sódio, potássio, cálcio, fósforo, ALT, FA e GGT e seus respectivos testes estatísticos, nos momentos $\mathrm{PRH}$ e $\mathrm{POH}$. Os valores das médias de ureia apresentaram diferença estatística, sendo que os valores no $\mathrm{PRH}$ foram maiores que no $\mathrm{POH}(\mathrm{P}<0,001)$, assim como quando estudada a creatinina, onde houve diferença estatística entre os momentos, sendo que os valores PRH foram maiores que $\mathrm{POH}(\mathrm{P}<0,005)$. Os valores de sódio,

Quadro 2. Mediana, semi-amplitude interquantílica e valores mínimo e máximo do número de hemácias, volume globular e plaquetas nos momentos pré e pós hemodiálise e resultado do teste estatístico da comparação de momentos

\begin{tabular}{|c|c|c|c|c|c|c|}
\hline \multirow{3}{*}{$\begin{array}{c}\text { Medidas } \\
\text { descritivas }\end{array}$} & \multicolumn{6}{|c|}{ Avaliações } \\
\hline & \multicolumn{2}{|c|}{$\begin{array}{l}\text { Hemácias } \\
(\mu L)\end{array}$} & \multicolumn{2}{|c|}{$\begin{array}{c}\text { Volume } \\
\text { globular (\%) }\end{array}$} & \multicolumn{2}{|c|}{$\begin{array}{l}\text { Plaquetas } \\
(\mu \mathrm{L})\end{array}$} \\
\hline & Pré & Pós & Pré & Pós & Pré & Pós \\
\hline Mediana & 3.970 .000 & 3.447 .000 & 28 & 23,1 & 135.903 & 45.723 \\
\hline $\begin{array}{l}\text { Semi-amplitude } \\
\text { interquartílica }\end{array}$ & 284.000 & 249.000 & 1,8 & 2,6 & 65.464 & 28.974 \\
\hline Valor máximo & 4.744 .000 & 4.118 .000 & 34,4 & 32 & 464.760 & 127.120 \\
\hline Valor mínimo & 3.346 .000 & 1.960 .000 & 23,6 & 14,4 & 39.410 & 35.700 \\
\hline
\end{tabular}

Quadro 3. Média e desvio padrão da determinação de hemoglobina, proteína total do plasma e contagem global de leucócitos nos momentos pré e pós hemodiálise e resultado do teste estatístico da comparação de momentos

\begin{tabular}{ccccccc}
\hline Medida & \multicolumn{5}{c}{ Avaliações } \\
\cline { 2 - 7 } descritiva & $\begin{array}{c}\text { Hemoglobina } \\
(\mathrm{mg} / \mathrm{dL})\end{array}$ & $\begin{array}{c}\text { Proteína total } \\
(\text { plasma })\end{array}(\mathrm{g} / \mathrm{dL})$ & \multicolumn{2}{c}{$\begin{array}{c}\text { Leucócitos } \\
(\mu \mathrm{L})\end{array}$} \\
\hline & Pré & Pós & Pré & Pós & Pré & Pós \\
Média & 9,281 & 7,799 & 6,11 & 5,45 & 11.731 & 9.063 \\
Desvio-padrão & 0,867 & 1,398 & 0,39 & 0,48 & 2.906 & 2.611
\end{tabular}

Resultado de teste estatístico ("P-value")5,07 ( $\mathrm{P}<0,001) 4,98(\mathrm{P}<0,001)$ $3,33(\mathrm{P}<0,005)$. 
Quadro 4. Mediana, semi-amplitude interquantílica e valores mínimo e máximo

das concentrações séricas de magnésio, glicose, proteína, albumina e globulina nos momentos pré e pós hemodiálise e resultado do teste estatístico da comparação de momentos

\begin{tabular}{|c|c|c|c|c|c|c|c|c|c|}
\hline \multirow{3}{*}{$\begin{array}{r}\text { Medidas } \\
\text { descritivas }\end{array}$} & \multicolumn{9}{|c|}{ Avaliações } \\
\hline & $\begin{array}{c}\text { Magnésio } \\
(\mathrm{mg} \%)\end{array}$ & \multicolumn{2}{|c|}{$\begin{array}{c}\text { Glicose } \\
(\mathrm{g} \%)\end{array}$} & \multicolumn{2}{|c|}{$\begin{array}{l}\text { Proteína } \\
\text { total }(g \%)\end{array}$} & \multicolumn{2}{|c|}{$\begin{array}{c}\text { Albumina } \\
(\mathrm{g} \%)\end{array}$} & \multicolumn{2}{|c|}{$\begin{array}{c}\text { Globulina } \\
(\mathrm{g} \%)\end{array}$} \\
\hline & $\overline{\text { Pré Pós }}$ & Pré & Pós & Pré & Pós & Pré & Pós & Pré & Pós \\
\hline Mediana & $1,5 \quad 1,5$ & 154,4 & 137,2 & 5,84 & 5,48 & 0,91 & 0,75 & 5 & 4,47 \\
\hline $\begin{array}{l}\text { Semi-amplitude } \\
\text { interquartílica }\end{array}$ & $0,370,11$ & 3,4 & 12,2 & 0,255 & 0,295 & 0,195 & 0,065 & 0,115 & 0,155 \\
\hline Valor máximo & $3,6 \quad 1,8$ & 180,6 & 157,6 & 8,94 & 8,94 & 1,48 & 0,84 & 6,02 & 7,22 \\
\hline Valor mínimo & $1,2 \quad 1,3$ & 135,8 & 84,6 & 5,34 & 5 & 0,82 & 0,54 & 4,58 & 3,48 \\
\hline Resultado de & 0,67 & \multicolumn{2}{|c|}{3,51} & \multicolumn{2}{|c|}{2,24} & \multicolumn{2}{|c|}{3,51} & \multicolumn{2}{|c|}{2.79} \\
\hline Teste estatístico & $(P>0,05)$ & \multicolumn{2}{|c|}{$(P<0,001)$} & \multicolumn{2}{|c|}{$(P<0,05)$} & \multicolumn{2}{|c|}{$(P<0,001)$} & \multicolumn{2}{|c|}{$(P<0,01)$} \\
\hline
\end{tabular}

Quadro 5. Média e desvio padrão da determinação de uréia, creatinina, sódio, potássio, cálcio, fósforo, ALT, FA e GGT nos momentos pré e pós hemodiálise e resultado do teste estatístico da comparação de momentos

\begin{tabular}{|c|c|c|c|c|c|}
\hline \multirow[t]{3}{*}{ Avaliações } & \multicolumn{5}{|c|}{ Medida descritiva } \\
\hline & \multicolumn{2}{|c|}{ Média } & \multicolumn{2}{|c|}{ Desvio-Padrão } & \multirow[t]{2}{*}{ (“P-value") } \\
\hline & Pré & Pós & Pré & Pós & \\
\hline Uréia (mg\%) & 35,79 & 15,85 & 11,99 & 3,44 & $5,80(P<0,001)$ \\
\hline Creatinina (mg\%) & 1,137 & 0,752 & 0,418 & 0,149 & $3,69(P<0,005)$ \\
\hline Sódio (mmol/L) & 143,72 & 143,94 & 2,55 & 1,43 & $0,32(P>0,05)$ \\
\hline Potássio (mmol/L) & 3,8 & 3.2 & 0,25 & 0,34 & $8,18(\mathrm{P}<0,001)$ \\
\hline Cálcio (mg\%) & 9,42 & 9,94 & 0,53 & 1,05 & $1,86(P>0,05)$ \\
\hline Fósforo (mg\%) & 4,6 & 3,9 & 0,95 & 0,63 & $2,56(P<0,05)$ \\
\hline ALT (U/L) & 40,77 & 43,40 & 7,58 & 8,76 & $0,93(P>0,05)$ \\
\hline $\mathrm{FA}(\mathrm{U} / \mathrm{L})$ & 109,2 & 126,6 & 43,46 & 31,67 & $1,26(P>0,05)$ \\
\hline GGT (U/L) & 5,47 & 5,48 & 1,73 & 1,48 & $0,02(P>0,05)$ \\
\hline
\end{tabular}

Quadro 6. Mediana, semi-amplitude interquantílica e valores mínimo e máximo das concentrações séricas de bilirrubina total, direta e indireta nos momentos pré e pós hemodiálise e resultado do teste estatístico da comparação de momentos

\begin{tabular}{|c|c|c|c|c|c|c|}
\hline \multirow{3}{*}{$\begin{array}{c}\text { Medidas } \\
\text { descritivas }\end{array}$} & \multicolumn{6}{|c|}{ Avaliações } \\
\hline & \multicolumn{2}{|c|}{$\begin{array}{c}\text { Bilirrubina } \\
\text { direta }(\mathrm{mg} / \mathrm{L})\end{array}$} & \multicolumn{2}{|c|}{$\begin{array}{c}\text { Bilirrubina } \\
\text { total (mg/L) }\end{array}$} & \multicolumn{2}{|c|}{$\begin{array}{c}\text { Bilirrubina } \\
\text { indireta (mg/L) }\end{array}$} \\
\hline & Pré & Pós & Pré & Pós & Pré & Pós \\
\hline Median & 0,02 & 0,04 & 0,06 & 0,08 & 0,05 & 0,05 \\
\hline $\begin{array}{l}\text { Semi-amplitude } \\
\text { Interquartílica }\end{array}$ & 0,01 & 0,0 & 0,015 & 0,013 & 0,01 & 0,023 \\
\hline Valor máxi & 0,03 & 0,07 & 0,09 & 0,09 & 0,06 & 0,81 \\
\hline Valor mínimo & 0,01 & 0,01 & 0,04 & 0,05 & 0,03 & 0,01 \\
\hline $\begin{array}{l}\text { Resultado de Teste } \\
\text { estatístico ("P-value") }\end{array}$ & \multicolumn{2}{|c|}{$\begin{array}{c}0,59 \\
(P>0,05)\end{array}$} & \multicolumn{2}{|c|}{$\begin{array}{c}2,48 \\
(P<0,05)\end{array}$} & \multicolumn{2}{|c|}{$\begin{array}{c}0,82 \\
(P>0,05)\end{array}$} \\
\hline
\end{tabular}

cálcio, ALT, FA e GGT não apresentaram diferença estatística entre os momentos estudados $(P>0,05)$, enquanto que as dosagens de potássio e fósforo mostraram diferença estatística entre os momentos, sendo que $\mathrm{PRH}$ foi maior que $\mathrm{POH}(\mathrm{P}<0,001)$ e $(\mathrm{P}<0,05)$, respectivamente.

Os valores de bilirrubina total, direta e indireta expressos por mediana no momento PRH estão representados no Quadro 6. Estatisticamente, não houve diferença entre os momentos $\mathrm{PRH}$ e $\mathrm{POH}$ para a bilirrubina total $(\mathrm{P}>0,05)$ e indireta $(P>0,05)$, enquanto que houve diferença entre os momentos, pois a bilirrubina direta $(P<0,05)$, cujos valores $\mathrm{PRH}$ foram menores que $\mathrm{POH}$.

As determinações de $\mathrm{pH}, \mathrm{PO}_{2}, \mathrm{PCO}_{2}, \mathrm{cHCO}_{3}, \mathrm{ctCO}_{2} \mathrm{e}$ $\mathrm{SO}_{2}$, ocorreram nos momentos M0, M90 e M180. Em relação ao $\mathrm{pH}$, a análise estatística mostrou que a mediana em $\mathrm{MO}$ foi menor que nos demais momentos estudados, e que em M90 e M180 não foram diferentes entre si $(P<0,005)$. Os valores das médias de $\mathrm{CHCO}_{3}$, estatisticamente, mostraram que no M0 foi menor que em M90 e M180, que por sua vez não apresentaram diferenças significantes entre si $(P<0,01)$. Quanto à concentração de $\mathrm{CO}_{2}$ a análise estatística mostrou que não houve diferenças significativas entre os vários momentos ( $\mathrm{M0}=\mathrm{M} 90=\mathrm{M} 180)(\mathrm{P}>0,05)$, assim como demonstrado no Quadro 7.

O Quadro 8 representa as determinações de $\mathrm{PO}_{2}, \mathrm{PCO}_{2}$ e $\mathrm{SO}_{2}$. Quanto à $\mathrm{PO}_{2}$, a análise estatística mostrou que a mediana em MO foi menor que nos demais momentos estudados, e que em M90 e M180 não foram diferentes entre si $(P<0,01)$. Os valores das medianas de $\mathrm{PCO}_{2}$ não apresentaram diferença significativa $(\mathrm{M} 0=\mathrm{M} 90=\mathrm{M} 180)(\mathrm{P}>0,05)$, enquanto que as medianas de $\mathrm{SO}_{2}$ mostraram que $\mathrm{MO}$ foi menor que nos demais momentos estudados, e que por sua vez M90 e M180 não diferiram entre si $(P<0,001)$.

O Quadro 9 demonstra os resultados relativos à pressão arterial sistólica (PAS) e pressão arterial diastólica (PAD) em $\mathrm{mmHg}$, referentes aos momentos M0, M5, M30, M60, M90, M120, M150 e M180. Quanto à PAS, a análise estatística mostrou que a mediana de M5 não apresentou diferença significante em relação ao $\mathrm{M} 180$, e que nestes dois momentos foram menores quando comparados ao M30, M60, M90, M120 e M150, que por sua vez não apresentaram

Quadro 7. Média e desvio padrão da determinação de $\mathbf{p H}$, $\mathrm{CHCO}_{3}$ e concentração de $\mathrm{CO}_{2}$ nos momentos pré, 90 e 180 minutos de hemodiálise e resultado do teste estatístico da comparação de momentos

\begin{tabular}{|c|c|c|c|c|c|c|c|}
\hline \multirow{3}{*}{$\begin{array}{l}\text { Avali- } \\
\text { ações }\end{array}$} & \multicolumn{7}{|c|}{ Medida descritiva } \\
\hline & \multicolumn{3}{|c|}{ Média } & \multicolumn{3}{|c|}{ Desvio-padrão } & \multirow[t]{2}{*}{ ("P-value") } \\
\hline & Pré & $90 \mathrm{~min}$ & $180 \mathrm{~min}$ & Pré & $90 \mathrm{~min}$ & $180 \mathrm{~min}$ & \\
\hline $\mathrm{pr}$ & & 7,411 & 7,406 & 0,028 & 0,041 & 0,044 & 6,2 \\
\hline $\mathrm{CHCO}_{3}$ & 21,116 & 22,342 & 22,551 & 1,49 & 0,991 & 1,59 & $5,28(\mathrm{P}<$ \\
\hline $\mathrm{ctCO}_{2}$ & 19,894 & 20,177 & 20,828 & 1,515 & 1,245 & 1,628 & $1,96(P>0,05)$ \\
\hline
\end{tabular}


Quadro 8. Mediana, semi-amplitude interquantílica e valores mínimo e máximo das concentrações de PO2, PCO2 e SO2 nos momentos pré, 90 e 180 minutos de hemodiálise e resultado do teste estatístico da comparação de momentos

\begin{tabular}{|c|c|c|c|c|c|c|c|c|c|}
\hline \multirow{3}{*}{$\begin{array}{c}\text { Medidas } \\
\text { descritivas }\end{array}$} & \multicolumn{9}{|c|}{ Avaliações } \\
\hline & \multicolumn{3}{|c|}{$\mathrm{PO}_{2}$} & \multicolumn{3}{|c|}{$\mathrm{PCO}_{2}$} & \multicolumn{3}{|c|}{$\mathrm{SO}_{2}$} \\
\hline & Pré & 90min & $180 \mathrm{~min}$ & Pré & 90min & $180 \mathrm{~min}$ & Pré & 90min & 180min \\
\hline Mediana & 7,78 & 107,91 & 99,97 & 37,89 & 36,56 & 36,56 & 95,69 & 97,38 & 97,42 \\
\hline $\begin{array}{l}\text { Semi-amplitude } \\
\text { Interquartílica }\end{array}$ & 4,7 & 10,94 & 16,02 & 2,08 & 2,39 & 1,68 & 0,75 & 0,62 & 0,65 \\
\hline Valor máximo & 116,72 & 160,82 & 138,52 & 43,84 & 44,20 & 40,22 & 97,08 & 99,06 & 98,74 \\
\hline Valor mínimo & 71,62 & 87,86 & 83,62 & 34,48 & 30,72 & 32,36 & 76,44 & 95,24 & 94,38 \\
\hline $\begin{array}{l}\text { Resultado de Teste } \\
\text { estatístico ("P-value") }\end{array}$ & $\begin{array}{r}0 \\
(\mathrm{P}>\end{array}$ & $\begin{array}{l}, 59 \\
0,05)\end{array}$ & $\begin{array}{c}3,13 \\
(P>0,05)\end{array}$ & $\begin{aligned} 1 \varepsilon \\
(P<\end{aligned}$ & $\begin{array}{l}, 38 \\
, 001)\end{array}$ & & & & \\
\hline
\end{tabular}

Quadro 9. Mediana, semi-amplitude interquantílica e valores mínimo e máximo das mensurações das pressões arteriais sistólica (PAS) e diastólicas (PAD) nos momentos pré, $5,30,60,90,120,150$ e 180 minutos de hemodiálise e resultado do teste estatístico da comparação de momentos

\begin{tabular}{|c|c|c|c|c|c|c|c|c|}
\hline \multirow[t]{3}{*}{ Avaliações } & \multicolumn{8}{|c|}{ Medidas descritivas } \\
\hline & \multicolumn{4}{|c|}{ Pressão arterial sistólica (PAS) } & \multicolumn{4}{|c|}{ Pressão arterial diastólica (PAD) } \\
\hline & Mediana & $\begin{array}{l}\text { Semi-amplitude } \\
\text { interquartílica }\end{array}$ & $\begin{array}{l}\text { Valor } \\
\text { máx }\end{array}$ & $\begin{array}{l}\text { Valor } \\
\text { mín }\end{array}$ & Mediana & $\begin{array}{l}\text { Semi-amplitude } \\
\text { interquartílica }\end{array}$ & $\begin{array}{l}\text { Valor } \\
\text { máx }\end{array}$ & $\begin{array}{l}\text { Valor } \\
\text { mín }\end{array}$ \\
\hline PRE & 109 & 16,7 & 129 & 11 & 74 & 9 & 125 & 8 \\
\hline 5 Minutos & 91,50 & 8,75 & 107 & 54 & 56,50 & 6,75 & 96 & 42 \\
\hline 30 Minutos & 99,50 & 6,75 & 109 & 60 & 63,50 & 6,5 & 101 & 52 \\
\hline 60 Minutos & 98,50 & 7 & 119 & 48 & 65 & 10 & 118 & 52 \\
\hline 90 Minutos & 97,50 & 6,25 & 124 & 45,2 & 71,50 & 12 & 115 & 51 \\
\hline 120 Minutos & 100 & 6 & 117 & 38,2 & 69 & 10 & 116 & 51 \\
\hline 150 Minutos & 96,50 & 8,75 & 115 & 39 & 71 & 10,75 & 99 & 44 \\
\hline 180 Minutos & 93 & 10,75 & 119 & 12 & 69,50 & 11,5 & 106 & 53 \\
\hline Resultado de & \multicolumn{4}{|c|}{ 30,61 $(P<0,001)$} & \multicolumn{4}{|c|}{$41,86(P<0,001)$} \\
\hline
\end{tabular}

diferenças entre si. Além disso, a mediana no M0 foi menor que em todos os demais momentos estudados $(P<0,001)$. Em relação à $P A D$, a mediana no $M 5$ foi menor que em $M 0$, M30, M60, M90, M120, M150 e M180, que não apresentaram diferença estatística entre si $(\mathrm{P}<0,001)$.

\section{DISCUSSÃO}

A ocorrência de insuficiência renal é um dos maiores problemas na clínica veterinária de pequenos animais, visto que é a maior causa de óbitos nesse tipo de paciente. Atualmente, o tratamento é baseado na terapia conservadora, que consiste na fluidoterapia e em cuidados nutricionais. Com isso, a sobrevida e a qualidade de vida são baixas, tanto para os pacientes, quanto para os proprietários, como citado por Langstone (2002).

Este estudo foi desenvolvido, haja vista a necessidade de mudar esta situação, ou seja, para que o modelo proposto pudesse ser utilizado na prática clínica, e também para servir como base para outros estudos experimentais, em animais e também em humanos, desde que o peso corporal dos animais utilizados no experimento é semeIhante ao de pacientes pediátricos humanos.

Durante a realização do trabalho, diversos aspectos foram abordados para o desenvolvimento da técnica hemodialítica em cães. Dentre eles podemos destacar o acesso vascular, o protocolo anestésico, as alterações hema- tológicas e bioquímicas, a heparinização, alterações hemogasométricas, e complicações decorrentes da realização do procedimento, como hipotensão, necessidade transfusional e ocorrência de óbitos.

O acesso vascular adotado foi o cateter de duplo lúmen de 8 french de diâmetro. Optou-se por instalá-lo na veia jugular externa direita, como descrito por Cowgill (1995) e por Cowgill \& Langstone (1996), porém, em alguns animais, houve necessidade de se utilizar outra via de acesso (veia jugular externa esquerda), sem, entretanto, ter sido observado qualquer imprevisto relacionado a esse tipo de posicionamento. Todos os animais foram radiografados após a instalação do cateter, para verificação do correto posicionamento do mesmo. Conseguiu-se fluxo sanguíneo adequado, semelhante ao descrito por Cowgill (1995), e nenhuma complicação relacionada à perviedade, ou seja, presença de coágulos nas vias do cateter ou fluxo insuficiente aconteceu, assim como relatado por Langstone (2002), que considera esse tipo de acesso vascular como o ideal pra realização de $\mathrm{HD}$ em pequenos animais.

O sucesso deste procedimento aconteceu, possivelmente, pelo fato de que cuidados técnicos foram tomados, destacando a realização de radiografias para confirmação do posicionamento do mesmo, além da correta heparinização interdiálise, já que em humanos, a perda da perviedade do cateter, a obstrução por coágulos e seu mau 
posicionamento, é um dos maiores problemas encontrados, ocasionando insuficiência de fluxo, e dificultando, desta forma, a realização das sessões de HD (Riella 1996).

No protocolo anestésico, utilizou-se cloridrato de levomepromazina, como medicação pré-anestésica, propofol como agente indutor e o mesmo fármaco, como agente anestésico geral. Com essa associação, pudemos observar que os animais permaneceram em plano anestésico adequado para a realização do procedimento dialítico, proporcionando, ainda, um retorno rápido e confortável para todos os pacientes, dado este concordante com Paddelford (1999). Vale ressaltar, que com este protocolo, não observamos qualquer redução abrupta ou significativa da pressão arterial, permanecendo os animais com pressão arterial relativamente constante, durante o procedimento.

O propofol foi o agente anestésico de escolha para este experimento por diversos fatores, como a manutenção da pressão arterial constante, metabolização e excreção extra-renal, além da possibilidade de realizá-lo em infusão contínua. Além disso, esse agente não apresenta os efeitos colaterais observados com outros agentes, como barbitúricos e opioides (Paddelford 1999).

Em relação à hematimetria, pudemos notar diminuição dos valores de hemácias, hemoglobina, volume globular, fato semelhante ao relatado por Cowgill \& Langstone (1996), e reafirmados por Elliott et al. (2000). Faz-se importante ressaltar, que os valores iniciais encontrados estavam abaixo dos parâmetros normais esperados para a espécie de acordo com Kaneko (1997), fato este, possivelmente explicado, por carências nutricionais que estes animais apresentavam e também pelo fato de que, durante a realização do procedimento hemodialítico, ocorreram perdas sanguíneas, através das linhas e também dos dialisadores, além da possibilidade de fenômenos hemolíticos, decorrentes da passagem do sangue pelo circuito extracorpóreo.

Porém, os valores do hematócrito pré-hemodiálise não contra indicaram a realização da diálise, ressaltando-se ainda, que pacientes humanos com insuficiência renal crônica apresentam valores de série vermelha abaixo dos limites normais, fortalecendo ainda mais a viabilidade da realização do procedimento, em pacientes com síndrome anêmica. Não houve necessidade transfusional, em nenhum animal do experimento.

Quanto à contagem de plaquetas, pudemos notar diminuição nos valores do número global dessas células, dado que pode ser creditado a uma série de fatores, dentre eles, a ocorrência de reação de bioincompatibilidade com a membrana utilizada (polissulfona), provocada pelo contato do sangue do paciente com a membrana do dialisador, que pode desencadear ativação do sistema complemento, ocasionando então trombocitopenia, como referido por Cowgill \& Langstone (1996).

Devemos ainda considerar que os animais deste experimento, como já citado anteriormente, não tinham suporte nutricional adequado, antes do início da pesquisa, e que a grande maioria deles apresentava contagem global de plaquetas dentro dos limites mínimos esperados para a espécie.
A diminuição no número global de leucócitos pode decorrer de uma série de fatores, como a presença de reação de bioincompartibilidade, através da ativação do complemento (via alternativa), resultando em neutropenia, como citado por Cowgill \& Langstone (1996). Vale ressaltar, que esta alteração é transitória e não têm consequências clínicas importantes, além disso, os valores permaneceram na faixa esperada para a espécie (Kaneko 1997).

Com o protocolo de heparinização intermitente, conseguimos manter os valores de TCA dentro dos níveis adequados, evitando-se assim, nos animais do experimento, tanto a heparinização insuficiente quanto a excessiva, resultado conflitante com o de Cowgill \& Langstone (1996) e Langstone (2002), que sugeriram que a manutenção da heparinização ideal somente seria possível com heparinização em infusão contínua. Hoppensteadt et al. (1999) utilizaram protocolo semelhante ao descrito neste estudo, diferindo apenas a dose do bolus inicial $(250 \mathrm{u} / \mathrm{Kg})$ e relataram a formação de coágulos após 150 minutos de HD, o que não foi observado em nenhum momento deste estudo. A obtenção da heparinização adequada permite múltiplos reúsos dos dialisadores, fato este que é um dos mais importantes para verificar-se a viabilidade econômica da hemodiálise em animais. Segundo Hertel et al. (2001), a possibilidade de reúso é um dos indicadores de heparinização ideal.

Para obtenção dos resultados, ora descritos, possivelmente houve contribuição da monitorização da coagulação através do TCA, método habitualmente utilizado em cirurgias com circulação extracorpórea e por alguns centros, para HD aguda.

Os exames bioquímicos não revelaram mudanças importantes durante e após a HD. Alguns pontos devem ser considerados, como a não ocorrência de variação nos níveis séricos de sódio, possivelmente decorrente da utilização do chamado perfil de sódio, indicado por Cowgill (1995), e cuja finalidade é não permitir que os níveis deste eletrólito diminuam durante a realização do procedimento hemodialítico, fato este que aconteceu neste experimento, ou seja, conseguiu-se a estabilização dos níveis séricos de sódio, evitando-se assim a ocorrência de hiponatremia e suas consequências como hipotensão, náusea, êmese, dentre outras.

Os dados relativos às concentrações pré e pós-dialíticas de ureia mostraram queda significante, sendo o valor pósdialítico, aproximadamente $45 \%$ do valor inicial. Esta redução é compatível com "clearance" adequado dessa molécula, em que pese a concentração sanguínea anterior à diálise, que ressalta eficácia do método hemodialítico na remoção de toxinas de pequeno peso molecular.

A utilização de banhos isentos de glicose é comum na rotina clínica de centros de HD. Há possibilidade, com a utilização deste tipo de banho, da ocorrência de hipoglicemia, o que não foi observado no presente estudo. Este acontecimento deve ser decorrente de adequada neoglicogênese durante o procedimento hemodialítico, como proposto para pacientes humanos (Riella 1996). Os dados do presente trabalho confirmam o mesmo fenômeno em cães normais, submetidos ao tratamento hemodialítico. 
Durante as sessões de HD, os animais foram mantidos com cateter inserido na artéria femoral, para que se obtivessem os dados de pressão arterial e fosse possível a coleta de sangue para hemogasometria. Hematomas e outras reações locais não foram observadas.

Os valores hemogasométricos iniciais encontrados foram compatíveis com os esperados para a espécie (Kaneko 1997). Os dados obtidos são concordantes com os de Hunt et al. (1984), que não observaram alterações significativas com a utilização de membranas de polissulfona. Bregman et al. (2001) relataram diminuição dos níveis de $\mathrm{PO}_{2}$, durante as sessões de HD, fato este não encontrado nesse experimento, pois os animais foram mantidos com suplementação de oxigênio, através de sonda nasal, para que se evitasse hipoventilação e consequente diminuição de $\mathrm{PO}_{2}$.

Outro ponto importante a ser abordado, em relação às alterações hemogasométricas, é a utilização do bicarbonato como solução tampão, já que este tipo de tampão apresenta uma série de vantagens, principalmente quando comparadas com o acetato, como a rápida correção da acidose, distúrbio comum em pacientes com IRC, não ocorrência de hipoxemia, vasodilatação, além da diminuição de perda de dióxido de carbono e bicarbonato através do banho, como citados por Ganss et al. (1992), possível determinante de hipoventilação.

Uma das maiores complicações durante a realização das sessões de HD é a presença de quadro hipotensivo, assim como descrito por Langstone (2002), principalmente porque uma grande quantidade de sangue, cerca de $30 \%$ do volume sanguíneo, dependendo do peso corporal, é removido do paciente, ao início de cada tratamento, para que o circuito extracorpóreo seja preenchido. Uma das maneiras de se evitar esta complicação é a reposição simultânea do volume perdido com solução isotônica de $\mathrm{NaCl}$ $0,9 \%$, na tentativa de se manter a pressão sanguínea estável. Este procedimento foi adotado neste experimento.

No presente trabalho utilizou-se perfil de sódio, como proposto por Cowgill \& Langstone (1996) e reafirmado por Langstone et al. (1996). Este procedimento possivelmente contribuiu para evitar a ocorrência de episódios hipotensivos, mantendo-se tanto a PAS quanto a PAD estáveis durante as sessões.

Dois animais vieram a óbito durante o desenvolvimento do trabalho. Devemos salientar que estes fatos aconteceram no período interdialítico e não intradialítico, sendo possível explicar a perda desses animais pelo fato de que os mesmos apresentaram quadro trombocitopênico importante, apesar de terem iniciado as sessões de diálise com valores de plaquetas dentro da normalidade. Além do que, devemos lembrar que todos os animais estavam heparinizados, o que pode ter agravado o quadro, mostrando a necessidade de se estudarem novos anticoagulantes para serem utilizados neste tipo de paciente durante as sessões de HD.

\section{CONCLUSÃO}

Os resultados obtidos no presente trabalho permitiram concluir que foi possível o desenvolvimento do modelo proposto, de modo satisfatório, com pequeno número de intercorrências técnicas e clínicas. Assim, mostrou-se a HD em animais, método viável e seguro, que poderá contribuir para o tratamento clínico da IR em cães e para o desenvolvimento de pesquisas experimentais.

Agradecimentos.- À Fundação de Amparo à Pesquisa do Estado de São Paulo (FAPESP) e Fundação para o Desenvolvimento da Unesp (FundUnesp) pelo auxílio financeiro concedido a esta pesquisa. A Baxter Co. por ceder a hemodialisadora, sem a qual, esta pesquisa não teria sido realizada.

\section{REFERÊNCIAS}

Boim M.A., Teixeira V.P.C. \& Schor N. 2000. Rim e compostos vasoativos, p.21-39. In: Zatz R. (Ed.), Fisiopatologia Renal. Atheneu, São Paulo.

Bregman H., Daugirdas J.T. \& Ing T.S. 2001. Complicações durante a hemodiálise, p.151-172. In: Daugirdas J.T., Blake P.G. \& Ing T.S. (Eds), Handbook of Hemodialysis. $3^{\text {rd }}$ ed. Little Brown, New York.

Cowgill L.D. 1995. Application of peritoneal dialysis and hemodialysis in the management of renal failure, p.573-579. In: Osborne C.A. \& Finco D.R. (Eds), Canine and Feline Nephrology and Urology. Williams and Wilkins, Baltimore.

Cowgill L.D. \& Langstone C. 1996. Role of hemodialysis in the management of dogs and cats with renal failure. Vet. Clin. North Am., Small Anim. Pract. 26:1347-1378.

Elliott D.A., Marks S.L., Cowgill L.D., Kass P.H. \& Rogers Q.R. 2000. Effect of hemodialysis on plasma amino acid concentrations in healthy dogs. Am. J. Vet. Res. 61(8):869-873.

Ganss R., Aarseth H.P. \& Nordby G. 1992. Prevention of hemodialysis associated hypoxemia by use of low-concentration bicarbonate dialysate. ASAIO J. 38(4):820-822.

Hertel J., Keep D.M. \& Caruana R.J. 2001. Anticoagulation, p.187-203. In: Daugirdas J.T., Blake P.G. \& Ing T.S. (Eds), Handbook of Hemodialysis. $3^{\text {rd }}$ ed. Little Brown, New York.

Hoppensteadt D.A., Jeske W.P. \& Walenga J.M. 1999. Laboratory monitoring of pentasaccharide in a dog model of Hemodialysis. Thromb. Res. 96:115-124.

Hunt J.M., Chappell T.R., Henrich W.L. \& Rubin L.J. 1984. Gas exchange during dialysis. Am. J. Med. 77:255-260.

Kaneko J.J. 1997. Clinical Biochemistry of Domestic Animals. Academic Press, San Diego, p.895-899.

Langstone C. 2002. Hemodialysis in dogs and cats. Compendium 24(7):540-548

Langstone C., Cowgill L.D. \& Spano J. 1996. The application of hemodialysis in uremis cats: A review of 24 cases. Proc. $14^{\text {th }}$ Annual Veterinary Medical Forum, San Antonio, p.750.

Meneses A.M.C., Caramori J.C.T., Brant J.R.A.C., Melchert A., Moutinho F.Q., Mamprim M.J. \& Barretti P. 2002. Acesso vascular para hemodiálise em cães utilizando cateter duplo lúmen. Anais XXIII Anclivepa, Brasília, DF, p.23. (Resumo).

Meneses A.M.C. 2003. Desenvolvimento de um modelo experimental de hemodiálise em cães (Canis familiaris). Dissertação de Mestrado em Fisiopatologia em Clínica Médica, Área de Concentração Nefrologia, Faculdade de Medicina de Botucatu, Universidade Estadual Paulista, Botucatu, SP. 93p.

Paddleford R.R. 1999. Manual of Small Animal Anesthesia. W.B. Saunders, Philadelphia, p.31-77.

Riella M.C. 1996. Princípios de Nefrologia e Distúrbios Hidroeletrolíticos. $3^{\text {a }}$ ed. Guanabara Koogan, Rio de Janeiro, p.89-110.

Siegel S. \& Castellan Jr N.J. 1988. Nonparametric Statistics on the Behavioral Sciences. $2^{\text {nd }}$ ed. McGraw Hill, New York. 399p.

Wichern D.W. \& Johnson R.A. 1992. Applied Multivariate Statistical Analysis. $6^{\text {th }}$ ed. Prentice Hall, New Jersey, p.173-174.

Yu L., Burdman E., Seguro A.C., Helou C.M.B. \& Zatz R. 2000. Insuficiência renal aguda, p.93-103. In: Zatz R. (Ed.), Fisiopatologia Renal. Atheneu, São Paulo. 